MATHEMATICS OF COMPUTATION

Volume 73, Number 246, Pages 1031-1037

S 0025-5718(03)01617-X

Article electronically published on August 20, 2003

\title{
SOME NEW KINDS OF PSEUDOPRIMES
}

\author{
JERZY BROWKIN
}

\begin{abstract}
We define some new kinds of pseudoprimes to several bases, which generalize strong pseudoprimes. We call them Sylow $p$-pseudoprimes and elementary Abelian $p$-pseudoprimes. It turns out that every $n<10^{12}$, which is a strong pseudoprime to bases 2, 3 and 5 , is not a Sylow $p$-pseudoprime to two of these bases for an appropriate prime $p \mid n-1$.

We also give examples of strong pseudoprimes to many bases which are not Sylow $p$-pseudoprimes to two bases only, where $p=2$ or 3 .
\end{abstract}

\section{INTRODUCTION}

The definition of strong pseudoprimes is based on the fact that in a finite field the equation $X^{2}=1$ has at most two solutions 1 and -1 . In the present paper we define more general pseudoprimes using a similar idea. In a finite field the equation $X^{r}=1$ has at most $r$ solutions, for every $r \geq 2$. Thus if for some $n$ the congruence $X^{r} \equiv 1(\bmod n)$ has more than $r$ solutions, then $n$ is composite. In our definition we consider several bases simultaneously to get many solutions of this congruence.

We give examples of strong pseudoprimes $n$ to several bases $b_{1}, \ldots, b_{k}$ which are not pseudoprimes in the new sense. In other words, no number $b_{j}(1 \leq j \leq k)$ is a witness for $n$ individually, but the set $\left\{b_{1}, \ldots, b_{k}\right\}$ is a witness for $n$; i.e., some properties of the set imply that $n$ is composite.

\section{Definitions}

Let $n>1$ be odd and let $p$ be a prime divisor of $n-1$. More precisely, let $n-1=p^{r} m$, where $r>0$ and $p \nmid m$. Let $b_{1}, \ldots, b_{k}$ be some residues modulo $n$ prime to $n$, and denote $a_{j}=b_{j}^{m}$, for $j=1, \ldots, k$.

If $n$ is a prime number, then $(\mathbb{Z} / n)^{*}$ is a cyclic group of order $n-1$. Consequently

(1) $b^{n-1}=1$, for every $b \in(\mathbb{Z} / n)^{*}$.

(2) The Sylow $p$-subgroup of $(\mathbb{Z} / n)^{*}$ is cyclic of order $p^{r}$.

(3) The maximal elementary Abelian $p$-subgroup of $(\mathbb{Z} / n)^{*}$ is cyclic of order $p$. In particular, for $p=2$, it is equal to $\{-1,1\}$.

(4) If $a$ is an element of $(\mathbb{Z} / n)^{*}$ of order $t>1$, then $1+a+a^{2}+\cdots+a^{t-1}=0$.

It follows that also the subgroup $G=\left\langle b_{1}, \ldots, b_{k}\right\rangle$ generated by the residues $b_{1}, \ldots, b_{k}$ is cyclic.

Hence

$\left(1^{\prime}\right) a_{j}^{p^{r}}=1$, for $j=1, \ldots, k$.

Received by the editor February 19, 1998 and, in revised form, October 23, 2002.

2000 Mathematics Subject Classification. Primary 11A15; Secondary 11A51, 11Y11.

Key words and phrases. Strong pseudoprimes, primality testing.

(C)2003 American Mathematical Society 
$\left(2^{\prime}\right)$ The Sylow $p$-subgroup of $G$ is cyclic of order dividing $p^{r}$.

$\left(3^{\prime}\right)$ The maximal elementary Abelian $p$-subgroup of $G$ is cyclic of order 1 or $p$. In particular, for $p=2$, it is a subgroup of $\{-1,1\}$.

$\left(4^{\prime}\right)$ For $1 \leq j \leq k$, if the order of $a_{j}$ is $p^{t}>1$, then

$$
1+a_{j}+a_{j}^{2}+\cdots+a_{j}^{p^{t}-1}=0 .
$$

Since the Sylow $p$-subgroup of $G$ is generated by $a_{1}, \ldots, a_{k}$, condition $\left(2^{\prime}\right)$ can be stated equivalently as

$\left(2^{\prime \prime}\right)$ If, say, $\operatorname{ord}\left(a_{1}\right) \geq \operatorname{ord}\left(a_{j}\right)$, for $j=1, \ldots, k$, then $a_{2}, \ldots, a_{k}$ belong to the group generated by $a_{1}$.

To reformulate $\left(3^{\prime}\right)$, we need the following notation. For $j=1, \ldots, k$, let

$$
c_{j}= \begin{cases}1, & \text { if } a_{j}=1, \\ a_{j}^{\operatorname{ord}\left(a_{j}\right) / p}, & \text { if } p \mid \operatorname{ord}\left(a_{j}\right) .\end{cases}
$$

Thus $c_{j}$ is an element of order 1 or $p$.

Evidently the maximal elementary Abelian $p$-subgroup of $G$ is generated by $c_{1}, \ldots, c_{k}$. Consequently $\left(3^{\prime}\right)$ can be stated equivalently as

$\left(3^{\prime \prime}\right)$ If, say, $\operatorname{ord}\left(c_{1}\right) \geq \operatorname{ord}\left(c_{j}\right)$, for $j=1, \ldots, k$, then $c_{2}, \ldots, c_{k}$ belong to the group generated by $c_{1}$. In particular, for $p=2$, every $c_{j}$ is equal to 1 or -1 .

Finally, from $\left(4^{\prime}\right)$ if follows

$\left(4^{\prime \prime}\right)$ If ord $c_{j}=p$, then $1+c_{j}+c_{j}^{2}+\cdots+c_{j}^{p-1}=0$.

The above properties of an odd prime number $n$ lead to the following definition.

\section{Definition.}

(i) We call a composite number $n$ satisfying $\left(1^{\prime}\right)$ a $p$-pseudoprime to bases $b_{1}, \ldots, b_{k}$. We use the notation $n \in p s p_{p}\left(b_{1}, \ldots, b_{k}\right)$.

(ii) We call a composite number $n$ satisfying $\left(1^{\prime}\right),\left(2^{\prime \prime}\right)$ and $\left(4^{\prime}\right)$ a Sylow $p$-pseudoprime to bases $b_{1}, \ldots, b_{k}$, and we use the notation

$$
n \in \operatorname{Syl}_{p}-\operatorname{psp}\left(b_{1}, \ldots, b_{k}\right) .
$$

(iii) We call a composite number $n$ satisfying $\left(1^{\prime}\right),\left(3^{\prime \prime}\right)$ and $\left(4^{\prime \prime}\right)$ an elementary Abelian $p$-pseudoprime to bases $b_{1}, \ldots, b_{k}$, and we use the notation

$$
n \in \text { Elem }_{p} \text {-psp }\left(b_{1}, \ldots, b_{k}\right) .
$$

In particular, elementary Abelian 2-pseudoprimes to bases $b_{1}, \ldots, b_{k}$ are strong pseudoprimes to these bases. Therefore in place of Elem ${ }_{2}-p s p\left(b_{1}, \ldots, b_{k}\right)$ we use the notation $\operatorname{spsp}\left(b_{1}, \ldots, b_{k}\right)$.

\section{REMARKS}

1. Condition $\left(2^{\prime \prime}\right)$ and condition $\left(3^{\prime \prime}\right)$ for $p>2$ are nontrivial only, if $k>1$, i.e., if we consider at least two bases. Conditions $(4),\left(4^{\prime}\right)$ and $\left(4^{\prime \prime}\right)$ give some information also if $k=1$.

2. Every Sylow $p$-pseudoprime to bases $b_{1}, \ldots, b_{k}$ is a fortiori an elementary Abelian $p$-pseudoprime to the same bases; thus

$$
\operatorname{Syl}_{p}-p s p\left(b_{1}, \ldots, b_{k}\right) \subset \text { Elem }_{p}-p s p\left(b_{1}, \ldots, b_{k}\right) \subset \operatorname{psp}_{p}\left(b_{1}, \ldots, b_{k}\right) \text {. }
$$

If moreover $p \| n-1$, then the first two sets are equal. 
3. If the set $\left\{c_{1}, \ldots, c_{k}\right\} \backslash\{1\}$ contains at least $p$ elements, then $\left(3^{\prime \prime}\right)$ is not satisfied, since in a cyclic group of order $p$ there are only $p-1$ elements of order $p$. Consequently $n$ is composite.

4. If an odd integer $n$ is Sylow $p$-pseudoprime or elementary Abelian $p$-pseudoprime, then $p \mid n-1$. Thus to prove that $n$ is not such a pseudoprime, we should consider prime divisors of $n-1$. The examples given in Tables 1 and 2 show that usually very small prime divisors $p$ of $n-1$ suffice, namely 2 , 3 or 5 with only one exception.

5. Evidently, if a positive integer $n$ satisfies (1) and does not satisfy at least one of the above conditions $(2)-\left(4^{\prime \prime}\right)$ for some prime divisor $p$ of $n-1$, then $n$ is composite. There are fast primality proving techniques available if $n-1$ is completely or even partially factored (see [KP] and [BLS]). Our examples suggest that the necessary information on prime divisors of $n-1$ can be further reduced.

\section{EXAMPLES}

We illustrate the above definitions by some known examples (see [J], [PSW]) of strong pseudoprimes $n$ to several bases $b_{1}, \ldots, b_{k}$. For some small prime divisors $p$ of $n-1$ and for bases $b_{1}, \ldots, b_{k}$ we write down the sequences

$$
b_{j}: a_{j}, a_{j}^{p}, a_{j}^{p^{2}}, \ldots, a_{j}^{p^{r}}, \text { for } j=1, \ldots, k .
$$

If $a_{j}^{p^{t}}=1$, for some $t$, we omit the next terms of the sequence since they are equal to 1 .

Example 1. Let $n=829 \cdot 1657=1373653$. Then $n-1=2^{2} \cdot 3^{3} \cdot 7 \cdot 23 \cdot 79$.

$\underline{p=2}$

$\overline{b_{1}=2}: a_{1}=890592, a_{1}^{2}=-1, \quad a_{1}^{4}=1$.

$b_{2}=3: a_{2}=1$.

Therefore $n$ is $\operatorname{spsp}(2,3)$ and even $n \in S y l_{2}-p s p(2,3)$.

$\underline{p=3}$

$\overline{b_{1}=2} \quad: \quad a_{1}=339686, \quad a_{1}^{3}=1168186, \quad a_{1}^{9}=1$.

$b_{2}=3 \quad: \quad a_{2}=220519, \quad a_{2}^{3}=1282588, \quad a_{2}^{9}=1$.

Therefore $c_{1}=a_{1}^{3}$, and $c_{2}=a_{2}^{3}$ are elements of order 3 . Since $c_{1}^{2}=1168186^{2} \equiv$ $210440 \not \equiv c_{2}(\bmod n)$, it follows that condition $\left(2^{\prime}\right)$ is not satisfied. Hence $n$ is composite, and $n$ is not Elem ${ }_{3}$-psp $(2,3)$. A fortiori it is not $\operatorname{Syl}_{3}-p s p(2,3)$.

Example 2. Let $n=4540612081 \cdot 9081224161=41234316135705689041$. Then $n-1=2^{4} \cdot 3^{3} \cdot \ldots$.

$p=2$

$\overline{b_{1}=2} \quad: \quad a_{1}=401 \ldots, \quad a_{1}^{2}=406 \ldots, \quad a_{1}^{4}=-1, \quad a_{1}^{8}=1$.

$b_{2}=3 \quad: \quad a_{2}=261 \ldots, \quad a_{2}^{2}=639 \ldots, \quad a_{2}^{4}=-1, \quad a_{2}^{8}=1$.

$b_{3}=5 \quad: \quad a_{3}=256 \ldots, \quad a_{3}^{2}=551 \ldots, \quad a_{3}^{4}=-1, \quad a_{3}^{8}=1$.

We have replaced last digits of large numbers by dots since these digits are not important for our purposes.

For $b_{j} \in\{7,11,13,17\}$ we have $a_{j}^{8}=-1$, and for $b_{9}=19$ we have $a_{9}^{4}=-1$.

Therefore $n$ is $\operatorname{spsp}(2,3,5,7,11,13,17,19)$.

Since $a_{1}^{2}, a_{2}^{2}, a_{3}^{2}$ are distinct elements of order four, the group generated by $b_{1}, b_{2}$, $b_{3}$ is not cyclic. Hence $n$ is composite and $n \notin S y l_{2}-p s p(2,3,5)$. 
Now, let $G$ be the group generated by $b_{1}$ and $b_{2}$ only.

One can easily verify that $\left(a_{1}^{2}\right)^{3}=a_{3}^{2} \neq a_{2}^{2}$. Hence in $G$ there are three distinct elements of order four: $a_{1}^{2}, a_{1}^{6}, a_{2}^{2}$. Consequently the group $G$ is not cyclic. Therefore in view of $\left(2^{\prime}\right) n$ is composite and $n \notin S y l_{2}-p s p(2,3)$.

We list the following numbers which are strong pseudoprimes to several bases (see [J]). The numbers $n_{1}$ and $n_{7}$ have been discussed in the above examples; we include them here for completeness.

$$
\begin{aligned}
& n_{1}=829 \cdot 1657=1373653 \\
& n_{2}=2251 \cdot 11251=25326001 \text {, } \\
& n_{1}-1=2^{2} \cdot 3^{3} \ldots \\
& n_{2}-1=2^{4} \cdot 3^{3} \cdot 5^{3} \ldots \\
& n_{3}=151 \cdot 751 \cdot 28351=3215031751 \text {, } \\
& n_{3}-1=2 \cdot 3^{4} \cdot 5^{3} \ldots \\
& n_{4}=6763 \cdot 10627 \cdot 29947=2152302898747 \text {, } \\
& n_{5}=1303 \cdot 16927 \cdot 157543=3474749660383 \text {, } \\
& n_{4}-1=2 \cdot 3 \cdot 7^{2} \ldots \\
& n_{6}=10670053 \cdot 32010157=341550071728321, \quad n_{6}-1=2^{6} \cdot 3 \cdot 5 \ldots \\
& n_{7}=4540612081 \cdot 9081224161=41234316135705689041 \text {, } \\
& n_{7}-1=2^{4} \cdot 3^{3} \ldots \\
& n_{8}=22754930352733 \cdot 68264791058197=1553360566073143205541002401 \text {, } \\
& n_{8}-1=2^{5} \cdot 3^{2} \cdot 5^{2} \ldots \\
& n_{9}=137716125329053 \cdot 413148375987157=56897193526942024370326972321 \text {, } \\
& n_{9}-1=2^{5} \cdot 3^{2} \cdot 5 \ldots
\end{aligned}
$$

\section{TABLES}

\begin{tabular}{|c|c|c|c|c|c|c|c|}
\hline$n$ & Is $s p s p$ to bases & \multicolumn{3}{|c|}{ Is $S y l_{p}-p s p$ ? } & \multicolumn{3}{|c|}{ 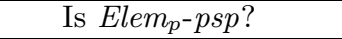 } \\
\hline & & $p$ & bases & & $p$ & bases & \\
\hline$n_{1}$ & 2,3 & 2 & 2,3 & YES & 3 & 2,3 & $\mathrm{NO}$ \\
\hline$\overline{n_{2}}$ & $2,3,5$ & 2 & $2,3,5$ & YES & 3 & 2,3 & $\mathrm{NO}$ \\
\hline$\overline{n_{3}}$ & $2,3,5,7$ & 2 & $2,3,5,7$ & \begin{tabular}{|c|c|} 
YES \\
\end{tabular} & $\overline{3}$ & $\overline{2,3}$ & $\mathrm{NO}$ \\
\hline$n_{4}$ & $2,3,5,7,11$ & 2 & $2,3,5,7,11$ & YES & 3 & 2,5 & $\mathrm{NO}$ \\
\hline$n_{5}$ & $2,3,5,7,11,13$ & 2 & $2,3,5,7,11,13$ & YES & 3 & 2,3 & $\mathrm{NO}$ \\
\hline$n_{6}$ & $2,3,5,7,11,13,17$ & 2 & 2,17 & $\mathrm{NO}$ & 3 & 3,5 & $\mathrm{NO}$ \\
\hline$\overline{n_{7}}$ & $2,3,5,7,11,13,17,19$ & & & & $\overline{2}$ & 2,3 & $\mathrm{NO}$ \\
\hline$n_{8}$ & $2,3,5,7,11,13,17,19,23$ & 2 & 2,11 & $\mathrm{NO}$ & 3 & 2 & $\mathrm{NO}$ \\
\hline$\overline{n_{9}}$ & $2,3,5,7,11,13,17,19,23,29$ & 2 & 2,7 & NO & 3 & 2,5 & $\mathrm{NO}$ \\
\hline
\end{tabular}

The above numbers $n_{j}$ satisfy (1) but do not satisfy some conditions of $\left(2^{\prime}\right)-\left(4^{\prime \prime}\right)$. Hence they are composite and are not elementary Abelian (respectively, Sylow) $p$ pseudoprimes to some bases, as is shown in Table 1. Let us remark that every number $n_{j}(j=1, \ldots, 9)$ does not belong to some $S y l_{p}-p s p\left(b_{1}, b_{2}\right)$, where $p=2$ or 3 and $b_{1}, b_{2} \in\{2,3,5\}$ are appropriate bases.

The computations have been done using the GP/PARI package, version 1.39.

We reproduce from [J] the list of all strong pseudoprimes $n<10^{12}$ to bases 2 , 3 and 5 . We have verified that for every $n$ in the list (with one exception) there exists a prime $p \in\{2,3,5\}$ and a basis $b_{1}, b_{2} \in\{2,3,5\}$ such that some of the conditions $\left(2^{\prime}\right)-\left(4^{\prime \prime}\right)$ are not satisfied. In some cases it is sufficient to consider only the one-element basis, when we use condition $\left(4^{\prime \prime}\right)$. It follows that $n$ is composite, and $n \notin S y l_{p}-p s p\left(b_{1}, b_{2}\right)$. The results are given in Table 2 .

The exceptional number $n$ (No. 73 in Table 2) satisfies $n-1=2^{2} \cdot 5 \cdot 13 \cdot \ldots$. Moreover, $n \in \operatorname{Syl}_{p}-p s p(2,3,5)$ for $p=2$ and 5 , but $n \notin p s p_{2}(13) \cup p p_{5}(13)$.

\section{TABLE 1.}


TABLE 2 .

\begin{tabular}{|c|c|c|c|c|}
\hline No. & $n$ & factorization of $n-1$ & $p$ & $b_{1}, b_{2}$ \\
\hline 1. & 25326001 & $2^{4} \cdot 3^{3} \cdot 5^{3} \cdot 7 \cdot 67$ & 3 & 2,3 \\
\hline 2. & 161304001 & $2^{6} \cdot 3 \cdot 5^{3} \cdot 11 \cdot 13 \cdot 47$ & 3 & 3,5 \\
\hline 3. & 960946321 & $2^{4} \cdot 3 \cdot 5 \cdot 29 \cdot 101 \cdot 1367$ & 2 & 2,5 \\
\hline 4. & 1157839381 & $2^{2} \cdot 3^{3} \cdot 5 \cdot 401 \cdot 5347$ & 3 & 3,5 \\
\hline 5. & 3215031751 & $2 \cdot 3^{4} \cdot 5^{3} \cdot 7 \cdot 37 \cdot 613$ & 3 & 2,3 \\
\hline 6. & 3697278427 & $2 \cdot 3^{3} \cdot 31 \cdot 563 \cdot 3923$ & 3 & 2,3 \\
\hline 7. & 5764643587 & $2 \cdot 3^{3} \cdot 13 \cdot 19 \cdot 37 \cdot 11681$ & 3 & 2 \\
\hline 8. & 6770862367 & $2 \cdot 3 \cdot 199 \cdot 827 \cdot 6857$ & 3 & 2,5 \\
\hline 9. & 14386156093 & $2^{2} \cdot 3^{3} \cdot 7 \cdot 11^{3} \cdot 17 \cdot 29^{2}$ & 2 & 2,5 \\
\hline 10. & 15579919981 & $2^{2} \cdot 3^{2} \cdot 5 \cdot 29 \cdot 1471 \cdot 2029$ & 3 & 2,3 \\
\hline 11. & 18459366157 & $2^{2} \cdot 3^{3} \cdot 7 \cdot 11 \cdot 17 \cdot 37 \cdot 3529$ & 3 & 2,3 \\
\hline 12. & 19887974881 & $2^{5} \cdot 3 \cdot 5 \cdot 19 \cdot 23 \cdot 59 \cdot 1607$ & 3 & 2 \\
\hline 13. & 21276028621 & $2^{2} \cdot 3^{4} \cdot 5 \cdot 7 \cdot 11 \cdot 19 \cdot 47 \cdot 191$ & 3 & 2,3 \\
\hline 14. & 27716349961 & $2^{3} \cdot 3^{4} \cdot 5 \cdot 13 \cdot 109 \cdot 6037$ & 2 & 3,5 \\
\hline 15. & 29118033181 & $2^{2} \cdot 3^{2} \cdot 5 \cdot 257 \cdot 313 \cdot 2011$ & 3 & 2,3 \\
\hline 16. & 37131467521 & $2^{8} \cdot 3 \cdot 5 \cdot 7 \cdot 73 \cdot 127 \cdot 149$ & 2 & 2,5 \\
\hline 17. & 41752650241 & $2^{9} \cdot 3^{2} \cdot 5 \cdot 7 \cdot 29 \cdot 79 \cdot 113$ & 2 & 2,5 \\
\hline 18. & 42550716781 & $2^{2} \cdot 3^{2} \cdot 5 \cdot 11 \cdot 13 \cdot 17 \cdot 97241$ & 3 & 2,3 \\
\hline 19. & 43536545821 & $2^{2} \cdot 3^{5} \cdot 5 \cdot 2459 \cdot 3643$ & 3 & 3,5 \\
\hline 20. & 44732778751 & $2 \cdot 3^{2} \cdot 5^{4} \cdot 11 \cdot 47 \cdot 7691$ & 3 & 2 \\
\hline 21. & 44778481441 & $2^{5} \cdot 3 \cdot 5 \cdot 7^{2} \cdot 11 \cdot 17 \cdot 10181$ & 3 & 2,5 \\
\hline 22 . & 48354810571 & $2 \cdot 3^{7} \cdot 5 \cdot 11 \cdot 19 \cdot 71 \cdot 149$ & 3 & 2,3 \\
\hline 23. & 52139147581 & $2^{2} \cdot 3^{4} \cdot 5 \cdot 13 \cdot 23 \cdot 107641$ & 3 & 2,3 \\
\hline 24. & 53700690781 & $2^{2} \cdot 3^{2} \cdot 5 \cdot 11 \cdot 2731 \cdot 9931$ & 3 & 2,3 \\
\hline 25. & 56209415767 & $2 \cdot 3 \cdot 7^{2} \cdot 23 \cdot 859 \cdot 9677$ & 3 & 2,5 \\
\hline 26. & 57698562127 & $2 \cdot 3 \cdot 37 \cdot 73 \cdot 541 \cdot 6581$ & 3 & 2,3 \\
\hline 27. & 67403434561 & $2^{6} \cdot 3 \cdot 5 \cdot 7 \cdot 11 \cdot 73 \cdot 12491$ & 2 & 2,5 \\
\hline 28. & 73796984161 & $2^{5} \cdot 3 \cdot 5 \cdot 79 \cdot 1307 \cdot 1489$ & 3 & 2,5 \\
\hline 29. & 74190097801 & $2^{3} \cdot 3^{3} \cdot 5^{2} \cdot 7 \cdot 13 \cdot 17 \cdot 83 \cdot 107$ & 3 & 2,3 \\
\hline 30. & 75285070351 & $2 \cdot 3 \cdot 5^{2} \cdot 7 \cdot 17 \cdot 269 \cdot 15679$ & 3 & 2,5 \\
\hline 31. & 75350936251 & $2 \cdot 3^{2} \cdot 5^{4} \cdot 19 \cdot 61 \cdot 5779$ & 3 & 2,3 \\
\hline 32 . & 77475820141 & $2^{2} \cdot 3^{3} \cdot 5 \cdot 163 \cdot 541 \cdot 1627$ & 3 & 2,3 \\
\hline 33. & 79696887661 & $2^{2} \cdot 3^{3} \cdot 5 \cdot 13 \cdot 29 \cdot 353 \cdot 1109$ & 3 & 2,5 \\
\hline 34. & 83828294551 & $2 \cdot 3^{3} \cdot 5^{2} \cdot 7 \cdot 11 \cdot 13 \cdot 17 \cdot 41 \cdot 89$ & 3 & 2 \\
\hline 35. & 88473676747 & $2 \cdot 3 \cdot 7 \cdot 13 \cdot 67 \cdot 683 \cdot 3541$ & 3 & 2,3 \\
\hline 36. & 88974090367 & $2 \cdot 3 \cdot 7 \cdot 53 \cdot 67 \cdot 596573$ & 3 & 2 \\
\hline 37. & 98515393021 & $2^{2} \cdot 3^{5} \cdot 5 \cdot 11 \cdot 137 \cdot 13451$ & 3 & 2 \\
\hline 38. & 111737197441 & $2^{7} \cdot 3^{3} \cdot 5 \cdot 11 \cdot 17 \cdot 151 \cdot 229$ & 3 & 2 \\
\hline 39. & 114247549027 & $2 \cdot 3^{2} \cdot 13 \cdot 41 \cdot 149 \cdot 229 \cdot 349$ & 3 & 2,3 \\
\hline 40. & 118670087467 & $2 \cdot 3^{2} \cdot 7 \cdot 47 \cdot 107 \cdot 137 \cdot 1367$ & 3 & 2 \\
\hline 41. & 126223730461 & $2^{2} \cdot 3^{5} \cdot 5 \cdot 53 \cdot 79 \cdot 6203$ & 5 & 2,3 \\
\hline 42. & 134670080641 & $2^{7} \cdot 3 \cdot 5 \cdot 61 \cdot 521 \cdot 2207$ & 2 & 2,3 \\
\hline 43. & 135586888951 & $2 \cdot 3 \cdot 5^{2} \cdot 17 \cdot 19^{2} \cdot 147289$ & 3 & 2,3 \\
\hline 44. & 136136947201 & $2^{9} \cdot 3 \cdot 5^{2} \cdot 1627 \cdot 2179$ & 2 & 2,3 \\
\hline 45. & 148600530541 & $2^{2} \cdot 3^{2} \cdot 5 \cdot 7 \cdot 11 \cdot 59 \cdot 181721$ & 3 & 3,5 \\
\hline 46. & 150401047441 & $2^{4} \cdot 3 \cdot 5 \cdot 7 \cdot 349 \cdot 256517$ & 3 & 2,3 \\
\hline
\end{tabular}


Table 2 (continued)

\begin{tabular}{|c|c|c|c|c|}
\hline No. & $n$ & factorization of $n-1$ & $p$ & $b_{1}, b_{2}$ \\
\hline 47. & 156677923729 & $2^{4} \cdot 3^{3} \cdot 19 \cdot 163 \cdot 181 \cdot 647$ & 3 & 2,3 \\
\hline 48. & 157615339681 & $2^{5} \cdot 3^{2} \cdot 5 \cdot 6367 \cdot 17191$ & 2 & 2,5 \\
\hline 49. & 167259489409 & $2^{7} \cdot 3^{5} \cdot 11 \cdot 433 \cdot 1129$ & 3 & 2,3 \\
\hline 50 . & 174460968067 & $2 \cdot 3 \cdot 7 \cdot 11 \cdot 19 \cdot 571 \cdot 34807$ & 3 & 2 \\
\hline 51. & 183413388211 & $2 \cdot 3 \cdot 5 \cdot 13 \cdot 31 \cdot 317 \cdot 47857$ & 3 & 2 \\
\hline 52. & 187403492251 & $2 \cdot 3^{2} \cdot 5^{3} \cdot 13 \cdot 37 \cdot 43 \cdot 4027$ & 3 & 2,3 \\
\hline 53. & 216291665041 & $2^{4} \cdot 3 \cdot 5 \cdot 11 \cdot 17 \cdot 47 \cdot 102539$ & 5 & 2,3 \\
\hline 54. & 218215348801 & $2^{6} \cdot 3 \cdot 5^{2} \cdot 29 \cdot 31 \cdot 61 \cdot 829$ & 3 & 3,5 \\
\hline 55. & 218673063181 & $2^{2} \cdot 3^{3} \cdot 5 \cdot 11 \cdot 13 \cdot 31 \cdot 167 \cdot 547$ & 3 & 2 \\
\hline 56. & 234311749201 & $2^{4} \cdot 3^{2} \cdot 5^{2} \cdot 53 \cdot 863 \cdot 1423$ & 2 & 2,3 \\
\hline 57. & 240438464197 & $2^{2} \cdot 3 \cdot 7 \cdot 29 \cdot 4831 \cdot 20431$ & 2 & 2,5 \\
\hline 58. & 244970876021 & $2^{2} \cdot 5 \cdot 13 \cdot 179 \cdot 521 \cdot 10103$ & 5 & 2,3 \\
\hline 59. & 245291853691 & $2 \cdot 3^{4} \cdot 5 \cdot 13 \cdot 17 \cdot 1370269$ & 3 & 2 \\
\hline 60. & 247945488451 & $2 \cdot 3 \cdot 5^{2} \cdot 11 \cdot 19 \cdot 43 \cdot 193 \cdot 953$ & 3 & 2 \\
\hline 61. & 252505670761 & $2^{3} \cdot 3^{4} \cdot 5 \cdot 7 \cdot 47 \cdot 236881$ & 2 & 2,5 \\
\hline 62. & 272447722207 & $2 \cdot 3^{6} \cdot 11 \cdot 179 \cdot 94903$ & 3 & 2 \\
\hline 63. & 291879706861 & $2^{2} \cdot 3^{2} \cdot 5 \cdot 7 \cdot 6367 \cdot 36383$ & 3 & 2 \\
\hline 64. & 295545735181 & $2^{2} \cdot 3^{4} \cdot 5 \cdot 137 \cdot 1331647$ & 3 & 2,3 \\
\hline 65. & 307768373641 & $2^{3} \cdot 3^{2} \cdot 5 \cdot 7 \cdot 13 \cdot 467 \cdot 20117$ & 2 & 2,5 \\
\hline 66. & 315962312077 & $2^{2} \cdot 3^{2} \cdot 37 \cdot 211 \cdot 479 \cdot 2347$ & 2 & 2,5 \\
\hline 67. & 331630652449 & $2^{5} \cdot 3^{2} \cdot 139 \cdot 193 \cdot 42923$ & 3 & 2,3 \\
\hline 68. & 342221459329 & $2^{7} \cdot 3^{4} \cdot 7 \cdot 757 \cdot 6229$ & 3 & 2,3 \\
\hline 69. & 353193975751 & $2 \cdot 3 \cdot 5^{3} \cdot 7 \cdot 11 \cdot 283 \cdot 21611$ & 3 & 2 \\
\hline 70. & 354864744877 & $2^{2} \cdot 3 \cdot 199 \cdot 5987 \cdot 24821$ & 3 & 2 \\
\hline 71. & 362742704101 & $2^{2} \cdot 3^{2} \cdot 5^{2} \cdot 7 \cdot 239 \cdot 240913$ & 3 & 2 \\
\hline 72 . & 398214876001 & $2^{5} \cdot 3 \cdot 5^{3} \cdot 97 \cdot 313 \cdot 1093$ & 2 & 2,5 \\
\hline 73. & 405439595861 & $2^{2} \cdot 5 \cdot 13 \cdot 47 \cdot 4999 \cdot 6637$ & - & - \\
\hline 74 . & 407979839041 & $2^{6} \cdot 3 \cdot 5 \cdot 79 \cdot 389 \cdot 13829$ & 3 & 3,5 \\
\hline 75. & 431229929521 & $2^{4} \cdot 3 \cdot 5 \cdot 13 \cdot 19 \cdot 37 \cdot 421 \cdot 467$ & 3 & 2,3 \\
\hline 76. & 457453568161 & $2^{5} \cdot 3^{2} \cdot 5 \cdot 10847 \cdot 29287$ & 2 & 2,5 \\
\hline 77. & 490883439061 & $2^{2} \cdot 3^{2} \cdot 5 \cdot 7 \cdot 23 \cdot 29 \cdot 359 \cdot 1627$ & 5 & 2,3 \\
\hline 78. & 503691743521 & $2^{5} \cdot 3^{3} \cdot 5 \cdot 7 \cdot 271 \cdot 61463$ & 3 & 2,3 \\
\hline 79. & 505130380987 & $2 \cdot 3 \cdot 7 \cdot 11 \cdot 8461 \cdot 129223$ & 3 & 2,3 \\
\hline 80. & 528929554561 & $2^{7} \cdot 3 \cdot 5 \cdot 11 \cdot 3181 \cdot 7873$ & 2 & 2,5 \\
\hline 81. & 546348519181 & $2^{2} \cdot 3^{3} \cdot 5 \cdot 19 \cdot 31 \cdot 281 \cdot 6113$ & 3 & 2 \\
\hline 82. & 549866444221 & $2^{2} \cdot 3^{4} \cdot 5 \cdot 101 \cdot 971 \cdot 3461$ & 3 & 2 \\
\hline 83. & 591090138721 & $2^{5} \cdot 3^{4} \cdot 5 \cdot 17 \cdot 137 \cdot 19583$ & 3 & 2 \\
\hline 84. & 641498618881 & $2^{10} \cdot 3^{2} \cdot 5 \cdot 7 \cdot 367 \cdot 5419$ & 3 & 2,3 \\
\hline 85. & 602248359169 & $2^{8} \cdot 3^{4} \cdot 4519 \cdot 6427$ & 3 & 2,3 \\
\hline 86. & 659937299407 & $2 \cdot 3 \cdot 7 \cdot 11 \cdot 19 \cdot 509 \cdot 147703$ & 3 & 2,5 \\
\hline 87. & 688529415421 & $2^{2} \cdot 3^{3} \cdot 5 \cdot 7 \cdot 11 \cdot 23 \cdot 127 \cdot 5669$ & 3 & 2 \\
\hline 88. & 712614969307 & $2 \cdot 3^{2} \cdot 67 \cdot 113 \cdot 131 \cdot 179 \cdot 223$ & 3 & 2,3 \\
\hline 89 & 729421133761 & $2^{6} \cdot 3^{2} \cdot 5 \cdot 11 \cdot 41^{2} \cdot 13697$ & 3 & 2 \\
\hline 90 & 733224429367 & $2 \cdot 3 \cdot 11 \cdot 13 \cdot 251 \cdot 499 \cdot 6823$ & 3 & 3,5 \\
\hline 91. & 736775510329 & $2^{3} \cdot 3^{3} \cdot 11 \cdot 13 \cdot 2237 \cdot 10663$ & 3 & 2 \\
\hline
\end{tabular}


Table 2 (continued)

\begin{tabular}{|l|c|l|l|l|}
\hline No. & $n$ & factorization of $n-1$ & $p$ & $b_{1}, b_{2}$ \\
\hline 92. & 741881186287 & $2 \cdot 3 \cdot 71777 \cdot 1722653$ & 3 & 2 \\
93. & 744049848481 & $2^{5} \cdot 3 \cdot 5 \cdot 41 \cdot 47 \cdot 883 \cdot 911$ & 3 & 2,3 \\
94. & 774840343681 & $2^{7} \cdot 3^{2} \cdot 5 \cdot 13 \cdot 19 \cdot 733 \cdot 743$ & 3 & 2 \\
95. & 842638521121 & $2^{5} \cdot 3 \cdot 5 \cdot 11^{2} \cdot 23 \cdot 73 \cdot 8641$ & 3 & 2 \\
96. & 851402588401 & $2^{4} \cdot 3^{3} \cdot 5^{2} \cdot 7 \cdot 13 \cdot 17 \cdot 131 \cdot 389$ & 3 & 2 \\
97. & 853196213761 & $2^{9} \cdot 3 \cdot 5 \cdot 11 \cdot 1091 \cdot 9257$ & 2 & 2,3 \\
98. & 863370140641 & $2^{5} \cdot 3 \cdot 5 \cdot 7 \cdot 29 \cdot 991 \cdot 8941$ & 3 & 2 \\
99. & 908201935681 & $2^{6} \cdot 3 \cdot 5 \cdot 13 \cdot 47 \cdot 439 \cdot 3527$ & 3 & 2 \\
100. & 966299321527 & $2 \cdot 3 \cdot 7 \cdot 11^{2} \cdot 677 \cdot 280859$ & 3 & 2,5 \\
101. & 997031384161 & $2^{5} \cdot 3 \cdot 5 \cdot 7 \cdot 6863 \cdot 43237$ & 2 & 2,5 \\
\hline
\end{tabular}

Note added in March 2002. In a recent paper by Zhang [ZZ there are given tables of all strong pseudoprimes $<10^{24}$ (of some special kinds) to at least the first nine prime bases. We have verified that all these pseudoprimes are not elementary Abelian $p$-pseudoprimes for some bases $b_{1}, b_{2} \in\{2,3,5\}$ and some prime $p \in\{2,3,5\}$ with one exception. The number $n$ (No. 36 in Table 1 in [ZZ]) is not Elem $_{2}$-psp $(2,7)$.

Note added in October 2002. M. Agrawal, N. Kayal and N. Saxena [AKS] on August 6, 2002, presented a deterministic polynomial time algorithm that determines if a positive integer is prime or composite. In view of this result the importance of pseudoprimes of different kinds, including those defined in the present paper, has been drastically reduced.

\section{ACKNOWLEDGMENT}

I thank the referees for valuable comments that helped to improve the paper and Zhenxiang Zhang for sending me files containing strong pseudoprimes from his paper.

\section{REFERENCES}

[AKS] M. Agrawal, N. Kayal, N. Saxena, PRIMES is in P, http://www.cse.iitk.ac.in.

[BLS] J. Brillhart, D.H. Lehmer, J.L. Selfridge, New primality criteria and factorizations of $2^{m} \pm 1$, Math. Comp. 29 (1975), 620-647. MR 52:5546

[J] G. Jaeschke, On strong pseudoprimes to several bases, Math. Comp. 61 (1993), 915-926. MR 94d:11004

[KP] S. Konyagin, C. Pomerance, On primes recognizable in deterministic polynomial time, The mathematics of Paul Erdős (R.L. Graham, J. Nesetril, eds.), vol. I, Springer, Berlin, 1997, pp. 176-198. MR 98a:11184

[PSW] C. Pomerance, J.L. Selfridge, S.S. Wagstaff, Jr., The pseudoprimes to $25 \cdot 10^{9}$, Math. Comp. 35 (1980), 1003-1026. MR 82g:10030

[ZZ] Zhenxiang Zhang, Finding strong pseudoprimes to several bases, Math. Comp. 70 (2001), 863-872. MR 2001g:11009

Institute of Mathematics, University of Warsaw, Ul. Banacha 2, PL-02-097 Warsaw, POLAND

E-mail address: bro@mimuw.edu.pl 